\title{
The loading domain of the erythromycin polyketide synthase is not essential for erythromycin biosynthesis in Saccharopolyspora erythraea
}

Antibacterial Discovery Research, Abbott Laboratories, D-47P AP9A, 100 Abbott Park Rd, Abbott Park, IL 60064, USA

\author{
Ana Pereda, Richard G. Summers, Diane L. Stassi, Xiaoan Ruan \\ and Leonard Katz
}

Author for correspondence: Ana Pereda. Tel: +1 847938 0554. Fax: +1 8479383403

e-mail: Ana.Pereda@abbott.com

\begin{abstract}
6-Deoxyerythronolide B synthase (DEBS) is a large multifunctional enzyme that catalyses the biosynthesis of the erythromycin polyketide aglycone. DEBS is organized into six modules, each containing the enzymic domains required for a single condensation of carboxylic acid residues which make up the growing polyketide chain. Module 1 is preceded by loading acyltransferase (AT-L) and acyl carrier protein (ACP-L) domains, hypothesized to initiate polyketide chain growth with a propionate-derived moiety. Using recombinant DNA technology several mutant strains of Saccharopolyspora erythraea were constructed that lack the initial AT-L domain or that lack both the AT-L and ACP-L domains. These strains were still able to produce erythromycin, although at much lower levels than that produced by the wild-type strain. In addition, the AT-L domain expressed as a monofunctional enzyme was able to complement the deletion of this domain from the PKS, resulting in increased levels of erythromycin production. These findings indicate that neither the initial AT-L nor the ACP-L domains are required to initiate erythromycin biosynthesis; however, without these domains the efficiency of erythromycin biosynthesis is decreased significantly. It is proposed that in these mutants the first step in erythromycin biosynthesis is the charging of KS1 with propionate directly from propionyl-CoA.
\end{abstract}

Keywords: Streptomyces, polyketide synthase, loading domain

\section{INTRODUCTION}

Polyketides are a large and diverse class of natural products that includes antibiotic, anticancer, antiparasite, pigment and immunosuppressant compounds produced principally by Streptomyces and related filamentous bacteria. Biosynthesis of polyketides occurs by a mechanism that is similar to fatty acid biosynthesis but with some differences (Hopwood \& Sherman, 1990; Katz \& Donadio, 1993; Molnar et al., 1993; Hutchinson \& Fujii, 1995). Both polyketide synthases (PKSs) and

Abbreviations: $A C P$, acyl carrier protein; $A C P-L, A C P$ of loading domain; AT, acyltransferase; AT-L, AT of loading domain; DEB, 6-deoxyerythronolide $B$; DEBS, DEB synthase; DH, dehydratase; ER, enoyl reductase; eryA, genes encoding DEB synthase; KR, $\beta$-ketoreductase; KS, $\beta$-ketoacyl ACP synthase; PK, polyketide; PKS, polyketide synthase; RBS, ribosome-binding site. fatty acid synthases catalyse decarboxylative condensations between a thioester-linked nascent carbon chain and short-chain fatty acid extender units. PKSs, however, are considerably more diverse in the reactions that they can catalyse. This includes the use of different starters (acetate, propionate, butyrate) and chain extender units (malonate, methylmalonate, ethylmalonate) at various steps. Moreover, PKSs vary the extent of processing of the $\beta$-carbon after every condensation. Whereas the condensations of fatty acid biosynthesis are typically followed by a trio of reactions ( $\beta$-ketoreduction, dehydration, enoylreduction) to give a fully saturated carbon chain, a PKS may employ some, all, or none of these reactions after each condensation, giving rise to a highly functionalized carbon chain (Hopwood \& Sherman, 1990).

Erythromycin, a broad-spectrum macrolide antibiotic produced by Saccharopolyspora erythraea, is composed 


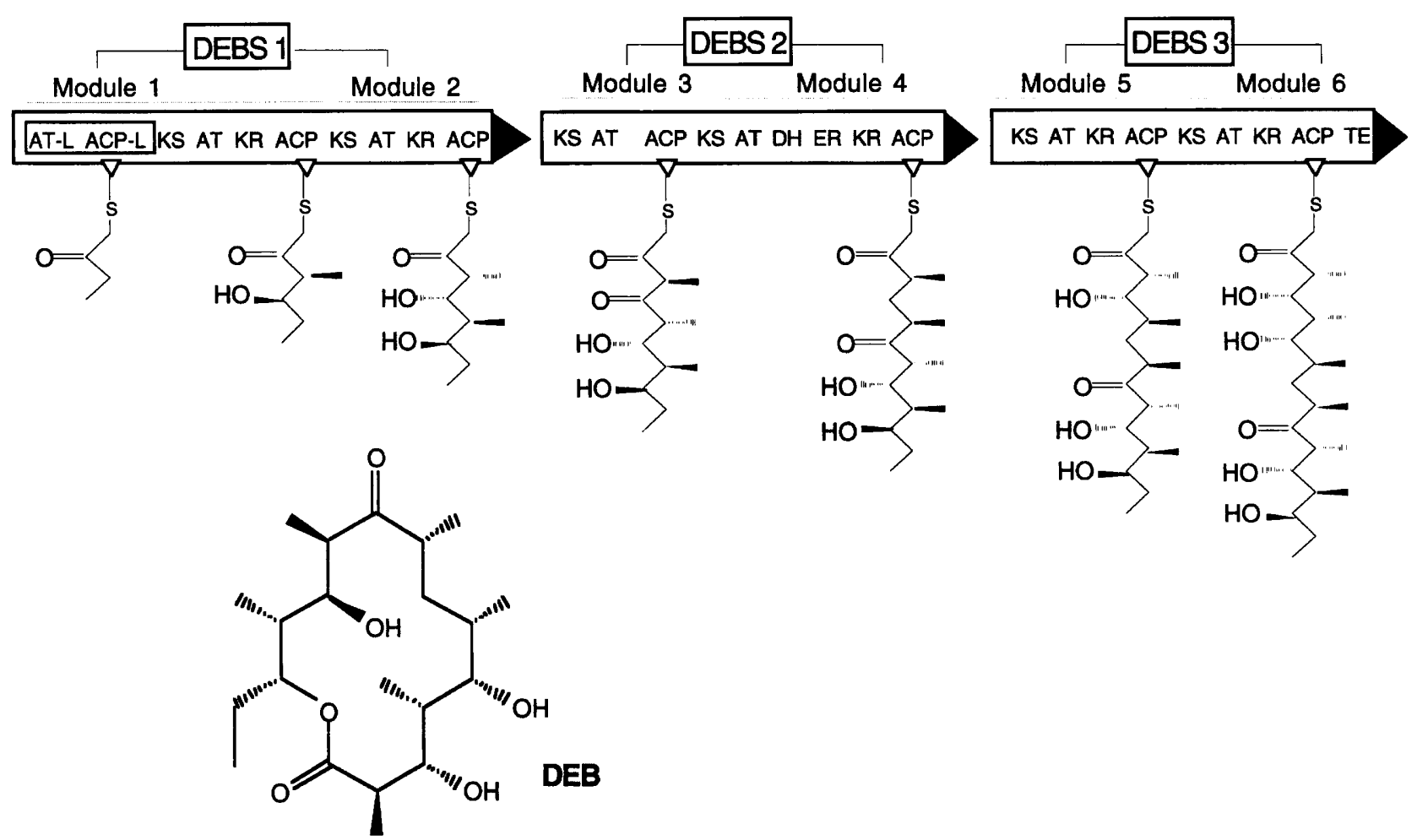

Fig. 1. Organization of the erythromycin PKS: 6-deoxyerythronolide $B$ (DEB) synthases 1,2 and 3 (DEBS1, 2, 3). The domains are designated as follows: acyltransferase, AT; $\beta$-ketoacyl carrier protein synthase, KS; acyl carrier protein, ACP; $\beta$-ketoreductase, KR; dehydratase, DH; enoyl reductase, ER; thioesterase,TE. The loading domain of module 1, comprising $A T-L$ and $A C P-L$, is boxed.

of a polyketide-derived macrocyclic lactone ring, 6deoxyerythronolide $\mathrm{B}$ (DEB), onto which two sugars, Lmycarose and D-desosamine, are attached at C-3 and C5 , respectively. The DEB core of erythromycin is derived from six successive condensations between a propionylCoA starter and six $(2 S)$-methylmalonyl-CoA extenders (Marsden et al., 1994). The DEB synthase (DEBS) is a multifunctional, or type I, PKS, encoded by three large genes, eryAI, eryAII and eryAIII (Cortes et al., 1990; Donadio et al., 1991; Donadio \& Katz, 1992; Caffrey et al., 1992; Bevitt et al., 1992). The three proteins encoded by the ery $A$ genes are organized in modules (Donadio $\&$ Katz, 1992; Ruby \& Danis, 1992; Swan et al., 1994; Schwecke et al., 1995). Each module contains the enzymic functions, as separate domains, for one of the six elongation cycles required for DEB formation. These include the $\beta$-ketoacyl-ACP synthase domain (KS), the acyltransferase domain (AT), and the acyl carrier protein domain (ACP). Also present may be $\beta$-ketoreductase (KR), dehydratase (DH) and enoyl reductase (ER) domains, responsible for the different degrees of reduction of the newly formed $\beta$-carbonyl group after each condensation step. In sum, DEBS comprises 28 enzymic activities, each dedicated to a single catalytic event (Fig. 1).

The PKS governs the selection of the starter molecule, the number and type of extender units added to the growing chain, and the extent and stereochemistry of reduction at each cycle, contributing to the structural variation found in polyketides. In modular PKSs it appears that the order of functional domains largely reflects the sequence of biochemical reactions (Donadio et al., 1991). Recently, the involvement of a distinct enzymic domain for each synthetic step has been exploited by genetically modifiying specific domains, thereby reprogramming the PKS to produce novel polyketide structures (Donadio et al., 1993; Olinynyk et al., 1996; Bedford et al., 1996; Kuhstoss et al., 1996; Ruan et al., 1997b).

The N-terminal end of module 1 of DEBS contains two additional enzymic domains, AT-L and ACP-L (Donadio et al., 1991). The AT-L domain is thought to initiate polyketide synthesis by choosing and transferring propionate from propionyl-CoA to the pantetheine cofactor of ACP-L. From here the propionate moiety is delivered to the active-site cysteine of the ketosynthase domain of module 1 (KS1). This work describes efforts to further define the constraints on PKS reprogramming by examining the role of the initial domains in the erythromycin PKS. Here we report the construction of a DEBS that lacks the initial acyltransferase domain and another in which both AT-L and the first acyl carrier protein, ACP-L, have been deleted. Both DEBS mutants still produce erythromycin A, although 
in lower yield than the wild-type. This suggests that the KS1 domain directly incorporates propionate from propionyl-CoA when the AT-L is absent in the mutant strains.

\section{METHODS}

Bacterial strains, plasmids and growth media. Saccharopolyspora erythraea ER720 (Dewitt, 1985) is the erythromycin-producing strain. Integrative transformation of $S$. erythraea protoplasts and routine growth and sporulation were carried out according to described procedures (Yamamoto et al., 1986; Donadio et al., 1991; Weber et al., 1991). Competent Escherichia coli DH5 $\alpha$ and the vectors pUC19, M13mp18 and M13mp19 were obtained from Bethesda Research Laboratories. The plasmid pSL1180 was purchased from Pharmacia Biotech. Plasmid pWHM3 (Vara et al., 1989) is a Streptomyces-E. coli shuttle vector that is poorly maintained episomally in Saccharopolyspora erythraea. Plasmid pWHM4 (Vara et al., 1989) is also a Streptomyces-E. coli vector that can replicate in Saccharopolyspora erythraea. Plasmids constructed for this study are listed in Table 1. For growth of S. erythraea strains in liquid culture, either SGGP medium (Yamamoto et al., 1986) or SCM medium (per litre of distilled $\mathrm{H}_{2} \mathrm{O}$ : soytone, $20 \mathrm{~g}$; soluble starch, $15 \mathrm{~g}$; MOPS, $10.5 \mathrm{~g}$; yeast extract, $1.5 \mathrm{~g} ; \mathrm{CaCl}_{2}, 0.1 \mathrm{~g}$ ) was used. For the growth of $S$. erythraea strains on plates, R3M was used [ 1 litre contains sucrose, $103 \mathrm{~g} ; \mathrm{K}_{2} \mathrm{SO}_{4}, 0.25 \mathrm{~g}$; yeast extract, $4 \mathrm{~g}$; Casamino acids, $4 \mathrm{~g}$; tryptone, $4 \mathrm{~g}$; agar, $22 \mathrm{~g}$; $\mathrm{H}_{2} \mathrm{O}, 830 \mathrm{ml}$; after sterilization the following solutions are added: $20 \mathrm{ml}$ $2.5 \mathrm{M} \mathrm{MgCl}_{2}, 20 \mathrm{ml} 50 \%$ glucose, $20 \mathrm{ml} 2.5 \mathrm{M} \mathrm{CaCl}_{2}, 12.5 \mathrm{ml}$

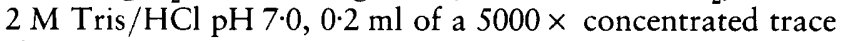
elements solution (Hopwood et al., 1985), $2.5 \mathrm{ml} 1 \mathrm{M} \mathrm{NaOH}$, and $\left.0.37 \mathrm{ml} 0.5 \mathrm{M} \mathrm{KH}_{2} \mathrm{PO}_{4}\right]$. When selection of thiostreptonresistant $S$. erythraea strains was required, $10 \mu \mathrm{g}$ thiostrepton $\mathrm{ml}^{-1}$ (Sigma) was used in liquid culture and $25 \mu \mathrm{g}$ thiostrepton $\mathrm{ml}^{-1}$ for growth on plates. Plasmid-containing E. coli strains were grown in Luria Broth (LB) (Sambrook et al., 1989) supplemented with $150 \mu \mathrm{g}$ ampicillin $\mathrm{ml}^{-1}$ (Sigma). For bioassays, antibiotic medium 11 (Difco-Bacto) containing Staphylococcus aureus as an indicator strain was used.

DNA manipulation. Plasmid isolations from E. coli, restriction digestion, ligation, agarose gel electrophoresis, recovery of DNA from agarose gels, and Southern hybridization were done as described by Sambrook et al. (1989). Restriction enzymes and T4 DNA ligase were purchased from BRL/Life Technologies or New England Biolabs. Plasmid and chromosomal DNA from $S$. erythraea were isolated according to Hopwood et al. (1985). The Amersham Megaprime DNA labelling system was used for labelling of DNA fragments with $\left[\alpha^{-32} \mathrm{P}\right] \mathrm{dCTP}$ (Amersham). For PCR, oligonucleotide primers (described in Table 2) were used with $S$. erythraea chromosomal DNA or pATD600 (Table 1) as the template. A typical PCR reaction to amplify the characteristically high GC content actinomycete DNA was as follows. A $100 \mu$ l reaction mixture contained $10 \mu \mathrm{l} 10 \times$ PCR buffer (New England Biolabs), $16 \mu \mathrm{l}$ $1.25 \mathrm{mM}$ dNTP mixture, $2 \mathrm{mM} \mathrm{MgSO}, 2 \%$ (v/v) glycerol, $10 \%$ formamide, $100 \mathrm{pmol}$ of each primer and $100 \mathrm{ng}$ template DNA. After 2 min incubation at $100^{\circ} \mathrm{C}, 2$ units Vent ${ }_{R}$ DNA Polymerase (New England Biolabs) was added, and then 30 cycles, each consisting of $20 \mathrm{~s}$ denaturation at $95^{\circ} \mathrm{C}, 20 \mathrm{~s}$ annealing at $67^{\circ} \mathrm{C}$ and a $2 \mathrm{~min}$ extension at $72{ }^{\circ} \mathrm{C}$, were performed in a GeneAmp PCR System 9600 (Perkin-Elmer Cetus).

Sequencing of the DNA fragments obtained by PCR was done with Sequenase 2.0 using 7-deaza-dGTP (United States Biochemical) according to the manufacturer's instructions and $\left[\alpha^{33} \mathrm{P}\right] \mathrm{dCTP}$. The Wisconsin Sequence Analysis Package program (Devereux et al., 1984) was used for sequence analysis.

Table 1. Plasmids used in this study

\begin{tabular}{|c|c|}
\hline Plasmid & Characteristics \\
\hline pATD600 & $\begin{array}{l}\text { A pSL1180 derivative containing a } 5 \cdot 8 \mathrm{~kb} \text { EcoRI-EcoRV fragment that includes } \\
\text { the codons for the entire N-terminal region of DEBS1 (the } 107 \text { aa segment, } \\
\text { AT-L, ACP-L, KS1 and a portion of AT1) }\end{array}$ \\
\hline pATD607 & $\begin{array}{l}\text { A pWHM3 derivative containing a } 4.45 \mathrm{~kb} \text { fragment that includes a portion of } \\
\text { the codons for the N-terminal region of DEBS1 (ACP-L, KS1 and the } \\
\text { beginning of AT1, but not the } 107 \text { aa segment or AT-L); used to construct } \\
\text { DEBS1-M1 }\end{array}$ \\
\hline pATD608 & $\begin{array}{l}\text { A pWHM3 derivative containing a } 4 \cdot 2 \mathrm{~kb} \text { fragment that includes a portion of } \\
\text { the codons for the N-terminal region of DEBS1 (KS1 and the beginning of AT1, } \\
\text { but not the } 107 \text { aa segment, AT-L or ACP-L); used to construct DEBS1-M2 }\end{array}$ \\
\hline pATD615 & $\begin{array}{l}\text { A pWHM3 derivative containing a } 4 \cdot 1 \mathrm{~kb} \text { fragment that includes a modified } \\
\text { portion of the N-terminal region of DEBS1 (eryKS1 replaced by nidKS1) but } \\
\text { lacks the codons for the } 107 \text { aa segment, AT-L and ACP-L; used to construct } \\
\text { DEBS1-M7 }\end{array}$ \\
\hline pATD622* & A pWHM4 derivative containing the $e r m E^{*}$ promoter \\
\hline pATD $623^{*}$ & $\begin{array}{l}\text { A pWHM4 derivative containing the erm } E^{*} \text { promoter and the codons for the } \\
107 \text { aa fragment plus the AT-L domain }\end{array}$ \\
\hline pATD624* & $\begin{array}{l}\text { A pWHM4 derivative containing the erm } E^{*} \text { promoter and the codons for the } \\
\text { AT-L domain }\end{array}$ \\
\hline
\end{tabular}

* These plasmids were used in the complementation experiments. 
Table 2. PCR primers designed for the construction of the plasmids used in this work

\begin{tabular}{|c|c|c|}
\hline No. & Description & Oligonucleotide sequence \\
\hline $\mathbf{I}$ & pATD607 & 5'-TTTTTTGAATTCACGCGTGGGCGTCGACTGGAACGC-3' \\
\hline & N-terminal & MluI M \\
\hline II & pATD608 & 5'-TTTTTTGAATTCACGCGTGGCACAACGGGAAACCCGC-3' \\
\hline & $\mathrm{N}$-terminal & MluI $\mathbf{M}$ \\
\hline III & pATD607-8 & 5'-TTTTTTGGATCCGAGCTCGAGCATGAGCCGCTGCTG-3' \\
\hline VI & $\begin{array}{l}\text { C-terminal } \\
\text { pATD614 }\end{array}$ & $\begin{array}{c}\text { BamHI Sst } \\
\text { 5'-TTTTTTGAATTCACGCGTGGTGTTCGTCTTCCCGGG-3' }\end{array}$ \\
\hline & N-terminal & MluI $\mathbf{M}$ \\
\hline VII & pATD614 & 5'-TTTTTTGGATCCACGCGTCCATCGCGGCCGCGAAAAC-3' \\
\hline & C-terminal & BamHI MluI \\
\hline VIII & $\begin{array}{l}\text { pATD622 } \\
\text { permE* } \text { N-terminal }^{-1}\end{array}$ & $\begin{array}{l}\text { 5'-TTTTTTAAGCTTGCGAGTGTCCGTTCGAGT-3' } \\
\text { HindIII }\end{array}$ \\
\hline IX & $\begin{array}{l}\text { pATD622 } \\
\text { permE* C-terminal }\end{array}$ & $\begin{array}{l}\text { 5'-TTTTTTCTGCAGCGCTGGATCCTACCAACC-3') } \\
\text { PstI }\end{array}$ \\
\hline $\mathbf{x}$ & pATD623 & 5'-TTTTTTGAATTCCTGCAGAGGAGGGCCTGCTCATGGCGGACCTGTCAAAG-3' \\
\hline & Upstream N-terminal & $\begin{array}{lll}E c o \text { RI } & P s t \mathrm{I} & \mathrm{RBS} \\
& & \end{array}$ \\
\hline XI & $\begin{array}{l}\text { pATD623-24 } \\
\text { Upstream C-terminal }\end{array}$ & $\begin{array}{l}\text { 5'-TTTTTTGGATCCACGCGTCCATCGCGGCGGCGA-3' } \\
\text { BamHI MluI }\end{array}$ \\
\hline XII & pATD624 & 5'-TTTTTTGAATTCCTGCAGAGGAGGGCCTGCTCATGGTGTTCGTCTTCCCGGGC-3' \\
\hline XIII & $\begin{array}{l}\text { Upstream N-terminal } \\
\text { pATD623-24 }\end{array}$ & $\begin{array}{cccc}\text { EcoRI } & \text { PstI } & \text { RBS } & \text { M }\end{array}$ \\
\hline XIII & $\begin{array}{l}\text { pA1D623-24 } \\
\text { Downstream } \mathrm{N} \text {-terminal }\end{array}$ & $\begin{array}{l}\text { EcoRI SphI } \\
\text { E }\end{array}$ \\
\hline XIV & $\begin{array}{l}\text { pATD623-24 } \\
\text { Downstream C-terminal }\end{array}$ & $\begin{array}{c}\text { 5'-TTTTTTGGATCCTCTAGATTAGCCGGACTCGGCCGGCTC-3' } \\
\text { BamHI } \quad \text { XbaI }\end{array}$ \\
\hline XVII & $\begin{array}{l}\text { pATD615 } \\
\text { nidKS1 N-terminal }\end{array}$ & $\begin{array}{l}\text { 5'-TTTTTTGAATTCACGCGTCGTCAACGGCGAGACC-3' } \\
E c o \text { RI } \quad M l u \mathrm{I}\end{array}$ \\
\hline & $\begin{array}{l}\text { I pATD615 } \\
\text { nidKS1 C-terminal }\end{array}$ & $\begin{array}{l}\text { 5'-TTTTTTGGATCCACGCGTGTT TCCCGTTGTGCGAC-3' } \\
\text { BamHI } \quad N s i \mathrm{I}\end{array}$ \\
\hline XIX & $\begin{array}{l}\text { pATD615 } \\
\text { eryAT1 N-terminal }\end{array}$ & $\begin{array}{c}\text { 5'-TTTTTTCTGCAGATGCATGCCATCATCGAGGAAGCT-3' } \\
\text { PstI } \quad N_{s i I}\end{array}$ \\
\hline $\mathbf{X X}$ & $\begin{array}{l}\text { pATD615 } \\
\text { eryAT1 C-terminal }\end{array}$ & $\begin{array}{l}\text { 5'-TTTTTTGGATCCGATATCCCTGCTCGGCGAGCGCAC-3' } \\
\text { BamHI EcoRV }\end{array}$ \\
\hline
\end{tabular}

Construction of DEBS mutant strains. Plasmids pATD607, pATD608, pATD614 and pATD615 (Table 1) were constructed to produce specific deletions at the beginning of the eryAI gene. To prepare these plasmids, segments of DNA upstream and downstream of the region to be deleted in eryAI were amplified by PCR, and the amplified fragments were then ligated together (using restriction enzyme sites in the PCR primers that maintain the correct translational reading frame) in plasmid $\mathrm{pWHM}$. Several deletions were constructed taking advantage of our current understanding of the organization of the DEBS1 protein. Translation of DEBS1 initiates 107 amino acids upstream of the first recognizable enzymic domain, ATL (Caffrey et al., 1992). AT-L is presumed to determine the choice of the starter unit for DEB biosynthesis (Donadio et al., 1991; Aparicio et al., 1994). In plasmid pATD607, the codons for all of AT-L plus the 107 aa upstream segment have been deleted (346 codons in total). To construct pATD607, an $M l u \mathrm{I}-S s t \mathrm{I}$ fragment (corresponding to nt 11540-12138: GenBank accession no. M63676) was PCR-amplified using primers I and III (Table 2). The resulting fragment was then used to replace the native $M l u I-S s t$ I fragment (nt 10174 12138: GenBank accession no. M63676) in the wild-type sequences of pATD600. Plasmid pATD608 is similar to pATD607, but also lacks the codons for ACP-L, thus a total of 421 codons have been deleted. To construct pATD608 another MluI-Sst I fragment (corresponding to nt 11798-12138: GenBank accession no. M63676) was PCR-amplified using primers II and III (Table 2) and then used to replace the wildtype sequences, as before. The initiating GTG codon at the beginning of DEBS1 overlaps the MluI restriction site used for these constructions; consequently, in both pATD607 and pATD608 the initiation codon is retained as is the natural ribosome-binding site. Plasmid pATD615 lacks the 107 aa segment, AT-L, and ACP-L from DEBS1 plus KS1 is replaced by nidKS1 from the Streptomyces caelestis PKS involved in the biosynthesis of niddamycin ( $\mathrm{S}$. Kakavas and others, unpublished). To make pATD615, nidKS1-encoding sequences were amplified by PCR using oligonucleotides XVII and XVIII 
(Table 2) as an MluI-NsiI fragment (corresponding to nt 2994-4285; S. Kakavas and others, unpublished). A segment downstream of eryKS1 was also amplified by PCR as an $N s i$-EcoR V fragment of 1121 bp (corresponding to nt $13107-$ 14228: GenBank accession no. M63676) using oligonucleotides XIX and XX. These two fragments were then assembled and used to replace an MluI-EcoRV fragment in pATD607. In all cases fidelity of the PCR reactions was confirmed by sequence analysis, and plasmids were verified by restriction analysis.

S. erythraea mutant strains were constructed by two sequential, plasmid-mediated, single reciprocal recombination events. First, integrative transformation based on homologous recombination of the $S$. erythraea chromosome with the plasmids described above was carried out as detailed by Weber \& Losick (1988) except that R3M plates were used for growth on solid media. Several of the resulting thiostreptonresistant transformants were then subjected to at least two rounds of non-selective growth in SGGP medium followed by protoplasting and plating. Single colonies were then screened for sensitivity to thiostrepton, indicating loss of the integrated plasmid by a second recombination event. The chromosomal DNA of the resulting thiostrepton-sensitive colonies was examined by Southern analysis (Sambrook et al., 1989). Between three and seven independent isolates in each case carried the appropriate mutation. Single isolates were then selected for further study and designated strains DEBS1-M1 (lacks AT-L and the upstream 107 aa segment), DEBS1-M2 (lacks the 107 aa segment, AT-L and ACP-L) and DEBS1-M7 (lacks the 107 aa segment, AT-L and ACP-L and KS1 is replaced by nidKS1).

Another mutant strain was created that lacked the 107 aa segment and AT-L, and in which the methylmalonyl-specific AT1 was replaced by a malonyl-specific AT from Streptomyces bygroscopicus (Ruan et al., 1997a). This mutant was constructed by transforming Saccharopolyspora erythraea EryAT1/'Hyg'AT2 (Ruan et al., 1997b) with plasmid pATD607 (Fig. 2), and has been designated DEBS1-M6 (lacks the 107 aa segment and AT-L, and AT1 is replaced by 'Hyg'AT2).

Construction of the AT-L expression plasmids. Plasmids pATD622, pATD623 and pATD624 (Table 1) were constructed to complement the DEBS1 deletion strains described above. All three plasmids contain the strong, constitutive ermE* promoter (Bibb et al., 1994). The promoter was obtained by PCR amplification from pIJ4070 using primers VIII and IX (Table 2), which introduce HindIII and PstI sites that allow easy transfer into pWHM4. Plasmid pATD622 carries just the ermE* promoter and was used as a control. Plasmid pATD623 resembles pATD622, but also includes a Pst $\mathrm{I}-\mathrm{X}$ bal segment carrying the DEBS1 ribosome-binding site, the 107 aa segment and AT-L. To minimize the occurrence of PCR-induced errors, this PstI-XbaI insert was assembled from three pieces. The $5^{\prime}$ and $3^{\prime}$ ends of the fragment were generated by PCR using primers X-XI and XIII-XIV, respectively (Table 2), with pATD600 as the template. These short fragments $(440 \mathrm{bp}$ and $140 \mathrm{bp}$ ) were then joined to the central MluI-SphI fragment $(810 \mathrm{bp})$ that was obtained from pATD600. Plasmid pATD624 is similar to pATD623 but it lacks the sequence encoding the 107 aa segment. In this case, PCR with primers XII-XI was used to generate a $110 \mathrm{bp}$ fragment that replaces the $440 \mathrm{bp}$ fragment described above in the construction of pATD623 (Table 2). Note that primers X and XII include the DEBS1 RBS and the GTG initiation codon while primer XIV introduces a TAA stop codon. In pATD623 and pATD624 this stop codon is presumed to terminate translation of the AT-L domain. The position of this stop codon corresponds exactly to the extent of the deletion in strain DEBS-M1. Each of the plasmids is maintained in S. erythraea by thiostrepton selection. The fidelity of the PCR-amplified fragments was confirmed by sequencing.

Metabolite identification. Cultures of S. erythraea ER 720 and the mutant strains, grown in SCM or SGGP for $3-4 \mathrm{~d}$ at $30^{\circ} \mathrm{C}$, were centrifuged for $5 \mathrm{~min}$ at 3500 r.p.m. The supernatants were adjusted to $\mathrm{pH} 9 \cdot 0$ with concentrated $\mathrm{NH}_{4} \mathrm{OH}$ and were then extracted twice with 1 vol. ethyl acetate. The organic phases were combined, concentrated by rotary evaporation, and analysed on silica gel TLC plates (Sigma-Aldrich). The plates were developed using isopropyl ether/methanol/ $\mathrm{NH}_{4} \mathrm{OH}(75: 35: 2$, by vol.), and anisaldehyde/sulfuric acid/ ethanol (1:1:9, by vol.) spray was used to visualize the compounds upon heating with a heat gun. For mass spectrometric analysis, the compounds were scraped from unstained TLC plates, and the portion of silica gel collected was extracted three times with ethyl acetate/methanol $(2: 1, \mathrm{v} / \mathrm{v})$ and concentrated by evaporation. Bioassays were performed by spotting dilutions of extracts of mutant and wild-type cultures onto a paper disc. The discs were then air dried and placed on a plate containing $100 \mathrm{ml}$ antibiotic medium 11 (Difco-Bacto) seeded with Staphyloccocus aureus as an indicator strain. A curve generated from dilutions of an erythromycin A standard was used to compare and evaluate the amount of compound produced by the mutant strains. The inhibition zones were developed by overnight incubation of the plate at $37^{\circ} \mathrm{C}$.

\section{RESULTS}

\section{The AT-L/ACP L deletion mutants are able to produce} low levels of erythromycin

The first polypeptide of the erythromycin PKS, DEBS1, has a 556 aa $\mathrm{N}$-terminal extension, the loading domain, that includes an acyltransferase (AT-L) and an acyl carrier protein (ACP-L). It has been proposed that the AT-L and ACP-L domains are involved in choosing the propionate starter unit for erythronolide chain growth (Donadio et al., 1991). Between the translation start site of DEBS1 and the beginning of the AT-L domain (defined by homology to type II soluble ATs) there is a stretch of $107 \mathrm{aa}$. This stretch precedes all AT domains in the DEBS1, DEBS2 and DEBS3 polypeptides, suggesting that the AT domains of a type I PKS may be larger than soluble type II AT enzymes. To examine the function of these elements in erythromycin biosynthesis, the N-terminus of DEBS1 was modified by introducing deletions into eryAI, the gene that encodes DEBS1. Plasmids which encode the $\mathrm{N}$-terminal region of DEBS1 lacking the 107 aa segment and AT-L only (pATD607), or the 107 aa segment, AT-L and ACP-L (pATD608) (Table 1) were constructed. These mutated segments were then used to replace their chromosomal counterparts in S. erythraea (see Methods), creating two strains: $S$. erythraea DEBS1-M1, whose PKS lacks the AT-L domain and the 107 aa upstream segment, and $S$. erythraea DEBS1-M2, whose PKS additionally lacks the ACP-L domain (Fig. 2).

Extracts of fermentation broths from each strain were analysed by TLC, bioassay (Fig. 3) and mass spec- 
(a)
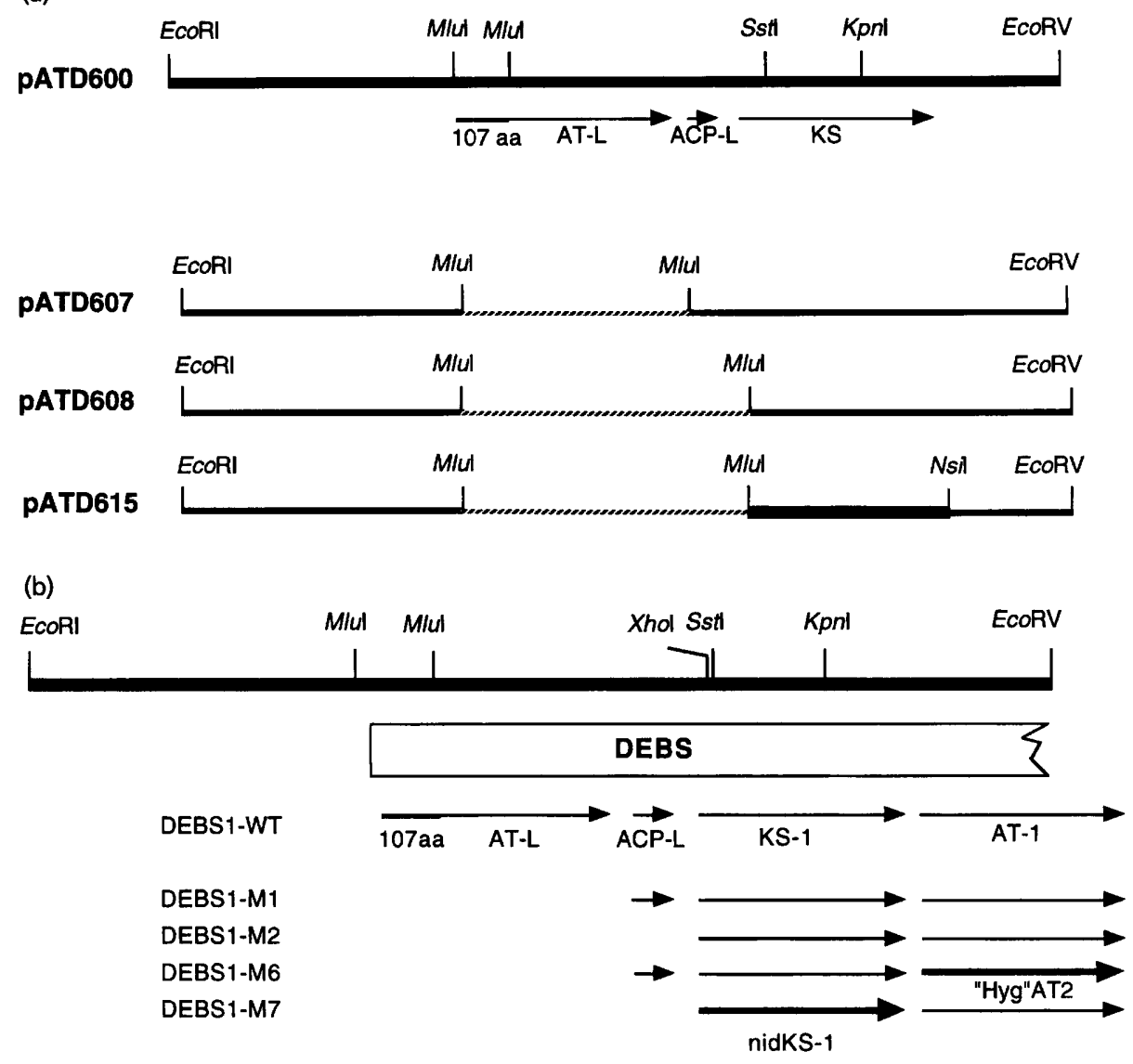

Fig. 2. Schematic representation of the plasmids (a) and mutant strains (b) generated in this study. The hatched regions in (a) indicate deleted segments.

trometry. Surprisingly, both strains continued to produce a bioactive compound that appeared to be erythromycin based on its $R_{\mathrm{F}}$ on TLC and its mass spectrometric profile. The yield in each case was low, however : both strains produced approximately $0.5 \%$ of the parental levels of erythromycin based on comparisons of inhibition zones produced by extracts of the mutant and parental strains (normalized for cell growth) (Table 3). Apparently, neither AT-L nor ACP-L is absolutely required for erythromycin biosynthesis, although they are important for efficient functioning of the PKS.

\section{A soluble form of AT- $L$ is able to partially restore erythromycin levels in the DEBS1-M1 mutant strain}

To determine whether the mutant DEBS1 polypeptides that lack their N-terminal domains could be complemented in vivo, two plasmids were constructed that express either the AT-L domain and the 107 aa segment, pATD623, or the AT-L domain without the 107 aa segment, pATD624 (Fig. 2a). These plasmids and an isogenic control that lacks any eryAI sequences (pATD622) were then transformed into strains DEBS1M1 and DEBS1-M2, and erythromycin A production of the transformants was assessed following 3 or $4 \mathrm{~d}$ growth. As shown in Fig. 4, strain DEBS1-M1 produced approximately 20 times more erythromycin A (Table 3 ) when the AT-L domain plus the 107 aa segment were provided in trans (pATD623), indicating that the soluble $\mathrm{N}$-terminal fragment of DEBS1 can still functionally associate with the truncated DEBS1 polypeptide and transfer propionate from propionyl-CoA to ACP-L. Interestingly, this association appears to require the 107 aa segment, since the AT-L domain lacking the 107 aa segment (pATD624) did not complement strain DEBS1M1 (Fig. 4), although we cannot rule out the possibility that the 107 aa segment is required for proper folding or enzymic activity of the AT-L domain. As expected, neither pATD623 nor pATD624 complemented strain DEBS1-M2 (Fig. 4), which lacks ACP-L, indicating that the ACP-L domain must be present for the DEBS1 polypeptide to accept propionate from AT-L.

\section{Initiation of erythromycin biosynthesis in the mutant strains occurs by direct loading of the KS1 domain}

Production of erythromycin A in mutant strains DEBS1M1 and DEBS1-M2 could occur in two ways. First, it is possible that the propionate starter for the initiation of 


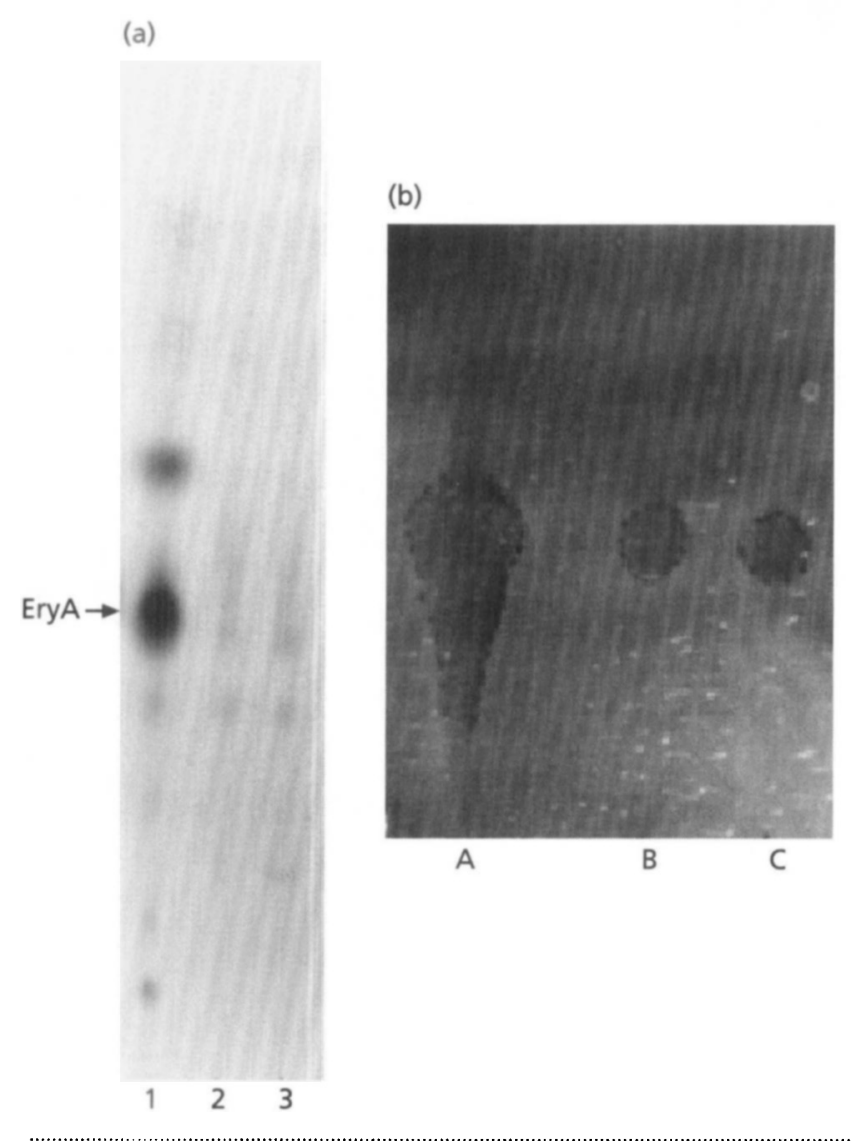

Fig. 3. TLC (a) and bioautography (b) of S. erythraea strains DEBS1-M1 and DEBS1-M2. (a) Lane1, DEBS1-WT; lane 2, DEBS1$\mathrm{M1}$; lane 3, DEBS1-M2; each lane corresponds to $5 \mathrm{ml}$ crude extract. (b) Lane A, DEBS1-WT; lane B, DEBS1-M1; lane C, DEBS1-M2; each lane corresponds to $100 \mu$ l crude extract.

erythromycin biosynthesis is loaded onto KS1 by the adjacent AT1 domain. Presumably, this would require decarboxylation of the methylmalonyl extender unit that is normally incorporated by AT1, followed by back-transfer to KS1. This hypothesis derives from the finding that the erythromycin PKS appears to catalyse decarboxylation of extender units and that the products of this reaction can then be used for polyketide biosynthesis in absence of the primer propionyl-CoA (Pieper et al., 1996a, b). The second possibility is that the propionate starter (from propionyl-CoA) is loaded

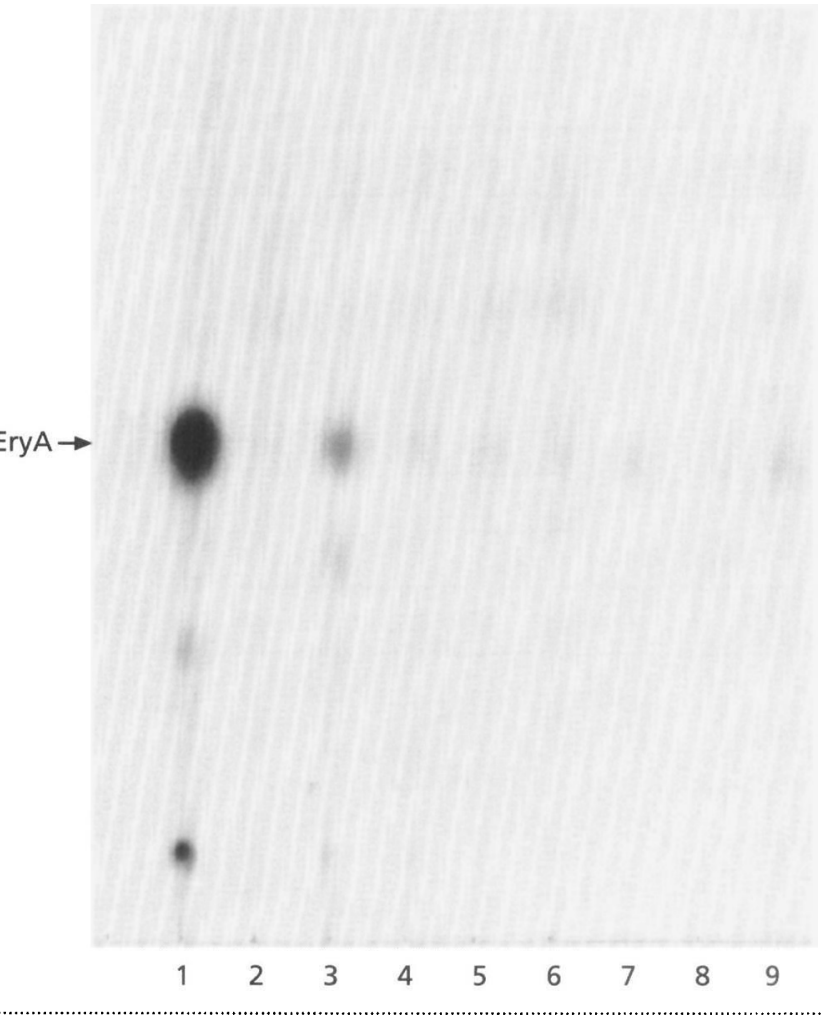

Fig. 4. TLC of ethyl acetate extracts from $5 \mathrm{ml}$ culture supernatants of $S$. erythraea strains DEBS1-M1 and DEBS1-M2 transformed with pATD622, pATD623 or pATD624. Lanes: 1, DEBS1-WT; 2, DEBS1-M1/no plasmid; 3, DEBS1-M1/pATD623; 4, DEBS1-M1/pATD624; 5, DEBS1-M1/pATD 622; 6, DEBS1-M2/no plasmid; 7, DEBS1-M2/pATD623; 8, DEBS1-M2/pATD624; 9, DEBS1-M2/pATD622.

directly onto the KS1 domain since the structure of the pantotheine thioester linkage of propionyl-ACP is identical to that of propionyl-CoA.

To distinguish between these alternatives, a new strain, DEBS1-M6 (Fig. 2), was constructed. DEBS1-M6 lacks both the 107 aa segment and AT-L domain (as in DEBS1M1) but also has had the methylmalonyl-specific eryAT1 domain replaced by the malonyl-specific 'Hyg'AT2 domain (Fig. 2) from a type I PKS cluster of the rapamycin producer Streptomyces hygroscopicus ATCC 29253 (Ruan et al., 1997a). Replacement of eryAT1 by 'Hyg'AT2 causes production of 12-

Table 3. Erythromycin production by the mutant strains alone and transformed with pATD622, pATD623 or PATD624

The values shown ( $\mu$ g erythromycin $\mathrm{ml}^{-1}$ ) are means of five experiments on different isolates. Fresh transformants were used for each experiment.

\begin{tabular}{|lcccc|}
\hline Strain & No plasmid & pATD622 & pATD623 & pATD624 \\
\hline DEBS1-WT & $25-35$ & $25-35$ & - & - \\
DEBS1-M1 & $0 \cdot 1-0 \cdot 3$ & $0 \cdot 1-0 \cdot 3$ & $3-5$ & $0 \cdot 1-0 \cdot 3$ \\
DEBS1-M2 & $0 \cdot 1-0 \cdot 4$ & $0 \cdot 1-0 \cdot 4$ & $0 \cdot 1-0 \cdot 4$ & $0 \cdot 1-0 \cdot 4$ \\
\hline
\end{tabular}




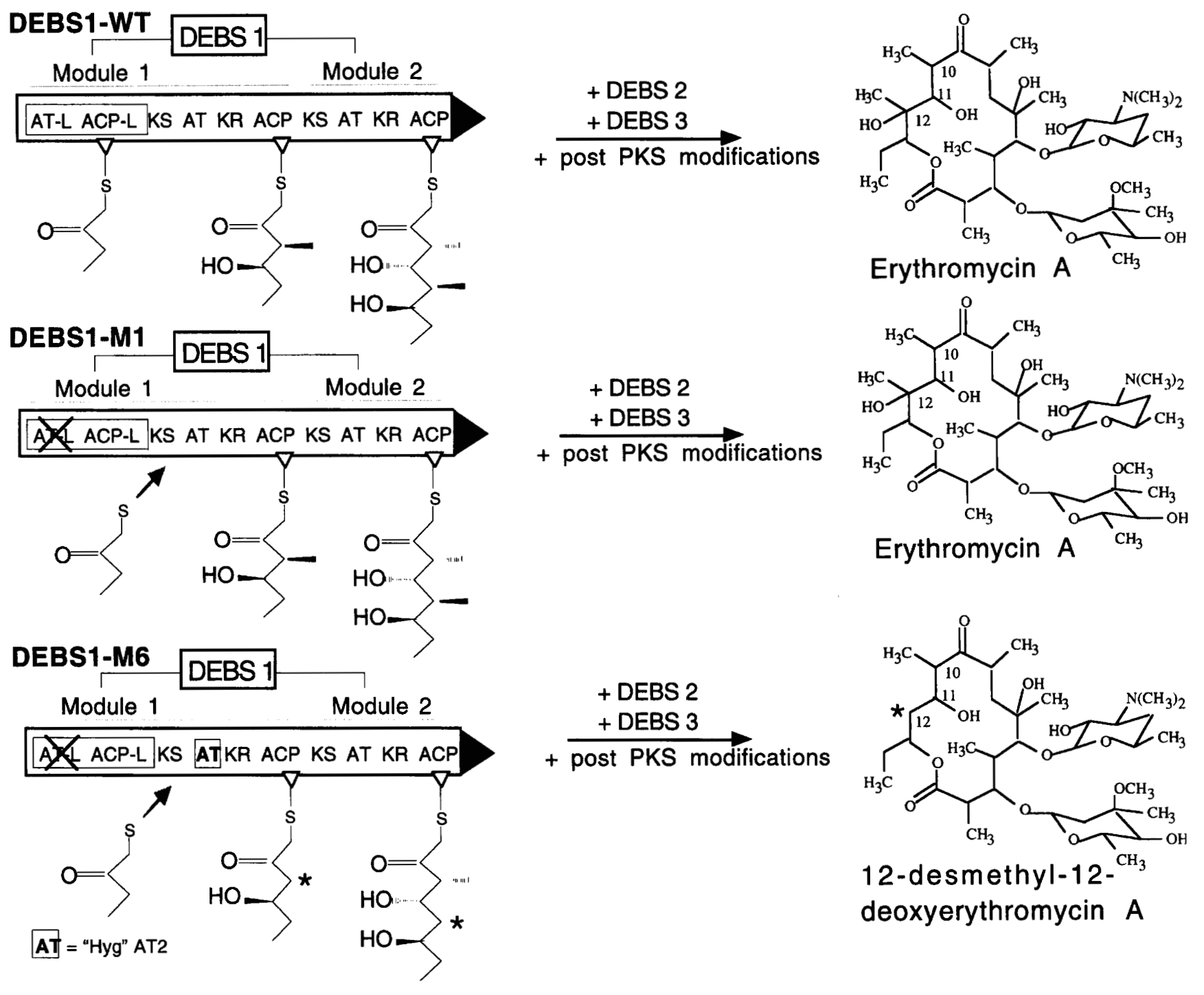

Fig. 5. Schematic representation of DEBS1 in the wild-type and S. erythraea strains DEBS1-M1 and DEBS1-M6. In DEBS1$M 1$, the AT-L domain has been deleted. In DEBS1-M6, the AT-L domain has been deleted and the natural AT1 domain has been replaced by a malonyl-CoA specific domain from Streptomyces hygroscopicus ('Hyg'AT2).

desmethyl-12-deoxyerythromycin A (Ruan et al., 1997b). If erythromycin synthesized by the AT-L-deleted DEBS1 strains is initiated by back-transfer (from AT1 to KS1) of a decarboxylated extender unit, then strain DEBS1-M6 should synthesize 12,14-didesmethyl-12deoxyerythromycin A (Fig. 5), due to back-transfer of an acetate moiety (i.e. decarboxylated malonate) from 'Hyg'AT2 to KS1. Alternatively, if KS1 loads propionate directly from propionyl-CoA, then strain DEBS1-M6 will make the same compound, 12desmethyl-12-deoxyerythromycin A, as the parent EryAT1/'Hyg'AT2 strain.

Mass spectrometric analysis of extracts obtained from fermentations of DEBS1-M6 revealed that this strain produces a compound of $m / z 704$, which corresponds to the molecular mass of 12-desmethyl-12-deoxyerythromycin A (Fig. 5). This indicates that back-transfer of decarboxylated extender units from AT1 to KS1 does not occur and suggests that KS1 loads propionate directly from propionyl-CoA. As was the case for the other AT-L-deleted strains, the level of compound produced by DEBS1-M6 is noticeably reduced, resembling that of DEBS1-M1.

Because strain DEBS1-M6 appears to load KS1 directly with a propionate starter molecule (Fig. 5), we decided to analyse the substrate preference of KS1 domains by constructing strain DEBS1-M7 (Fig. 2), in which the entire loading domain (107 aa segment, AT-L and ACPL) has been deleted and the eryKS1 domain is replaced by the nidKS1 domain from the niddamycin PKS cluster of Streptomyces caelestis (S. Kakavas and others, unpublished). Niddamycin is normally initiated with acetate from acetyl-CoA instead of propionate as in erythromycin. Consequently, the compounds produced 
by strain DEBS1-M7 should reveal whether the KS1 domains possess specificity for their appropriate starter. Interestingly, DEBS1-M7 continued to produce erythromycin as shown by mass spectrometric analysis of extracts, albeit in the low amounts characteristic of the N-terminally deleted DEBS1 strains. There was no evidence of erythromycin derivatives initiated with acetate, although we cannot exclude the possibility that some 15-norerythromycin $\mathrm{A}$ is made together with erythromycin A, but below the detection limits of this experiment. This result seems to suggest either that nidKS1 in strain DEBS1-M7 significantly prefers propionyl-CoA over acetyl-CoA or that propionyl-CoA levels in S. erythraea greatly exceed those of acetyl-CoA.

\section{DISCUSSION}

Erythromycin biosynthesis normally begins with the condensation of a coenzyme A thioester of a short-chain carboxylic acid 'starter unit', propionate, with a coenzyme A thioester of a dicarboxylic acid 'extender unit', methyl malonate. Partial proteolysis of DEBS has established that the N-terminal domain of DEBS1, corresponding to the loading domain, is specifically acylated by propionyl-CoA (Aparicio et al., 1994), indicating that this region of the PKS is responsible for choosing the starter molecule for erythromycin biosynthesis. This idea is further supported by the finding that the 'starter unit' specificity of the spiramycin PKS can be changed from acetate to propionate when its loading domain is replaced with the corresponding domain from the tylosin PKS (Kuhstoss et al., 1996). In this work, strains that lack the erythromycin PKS loading domain, in whole or in part, were constructed with the expectation that they would be incapable of producing erythromycin. Surprisingly, the deleted strains were still able to produce small amounts of erythromycin.

The KS domain in module 1 catalyses the condensation reaction between the starter and the extender incorporated by AT-L and AT1, respectively. One possible explanation for the continued synthesis of erythromycin in the deletion derivatives was that, in the absence of AT-L, AT1 can 'back-charge' KS1 with propionate (possibly formed from methylmalonate by decarboxylation). This does not appear to be the case, however, since strain DEBS1-M6 (which lacks AT-L and has had the methylmalonyl-specific AT1 replaced with a malonyl-specific AT) produces 12-desmethyl-12-deoxyerythromycin A. If back-charging had occurred, 12,14didesmethyl-12-deoxyerythromycin would have been the expected product, reflecting the use of acetate (from the new AT1) as both starter and the first extender. Consequently, it seems most likely that the biosynthesis of erythromycin by the mutants DEBS1-M1, DEBS1M2, DEBS1-M6 and DEBS1-M7 occurs by direct loading of propionate from propionyl-CoA onto the KS of module 1. (A similar, but more involved alternative would require direct loading onto KS1 of methylmalonate from methylmalonyl-CoA followed by de- carboxylation, but this seems less likely.) The substrate normally accepted by KS1 is propionyl-ACP, whose propionyl-pantotheine group is structurally identical to that of propionyl-CoA. This similarity may be sufficient to enable propionyl-CoA to bind productively at the KS1 active site. Consistent with this idea, direct acylation of DEBS with chain elongation intermediates (synthesized as their $\mathrm{N}$-acetylcysteamine derivatives, which also resemble the thioester portion of a phosphopantetheinylated molecule) has been demonstrated (Tsukamoto et al., 1996).

Interestingly, no compounds produced by these mutants using acetyl-CoA as a starter were detected. Kao et al. (1994) developed an engineered host-vector system for the expression of recombinant PKSs in Streptomyces coelicolor, and when this system was used to express the complete DEBS structural genes production of DEB and 8,8 a-deoxyoleandolide, which starts with acetate, was observed. It was suggested that the utilization of acetylCoA as a starter in this case could be due to the lower intracellular concentration of propionyl-CoA in the new host compared to the native erythromycin producer. $8,8 \mathrm{a}$-Deoxyoleandolide has also been isolated as a minor component produced by a gene-disrupted Saccharopolyspora erythraea strain (Weber et al., 1991). This demonstrates that DEBS can use acetate as starter in place of the normal propionate, although that does not seem to have taken place here even in the absence of ATL. Recent in vitro work has demonstrated that DEBS1 has a 32 -fold preference for a propionate starter over acetate and a 7.5 -fold preference for propionate over butyrate (Pieper et al., 1996). Moreover, S. erythraea appears to produce propionyl-CoA through the activity of a malonyl-CoA decarboxylase (Hsieh \& Kolattukudy, 1994), a fact that may indicate that the pool size of propionyl-CoA in S. erythraea could be larger than the pool size of acetyl-CoA. Whatever the reason, in the absence of the loading domain the mutants generated in this work clearly utilize propionyl-CoA preferentially.

To determine if KS1 is involved in determining specificity for propionate in these mutants we constructed strain DEBS1-M7, in which the natural KS1 has been replaced by the niddamycin KS1 (this strain also lacks the loading domain). Niddamycin synthesis normally initiates with acetyl-CoA. Unexpectedly, the DEBS1-M7 mutant produced erythromycin A instead of 14-desmethylerythromycin, the compound expected if the nidKS1 showed selectivity for acetyl-CoA. It seems that KS1 domains show some flexibility in the acyl chains that they will accept from a pantotheine donor, although in the context of $S$. erythraea the preference seems to be propionate over acetate. This may be because the concentration of propionyl-CoA greatly exceeds that of acetyl-CoA within the cell or because both the erythromycin and niddamycin KS1 domains bind propionyl-CoA more productively.

The levels of erythromycin synthesized by the AT-L deletion mutant DEBS1-M1 can be increased, although not to wild-type levels, by transforming the strain with 
pATD623, a multicopy plasmid that expresses the 107 aa segment and AT-L driven by the ermE $E^{*}$ promoter. This suggests that it is possible to associate a soluble AT enzymic domain with the multienzyme PKS and it opens new possibilities for introducing different soluble AT domains to create new erythromycin derivatives. As expected, the levels of erythromycin in mutant DEBS1M2, which lacks the 107 aa segment, AT-L and ACP-L, are not affected by the presence of plasmid pATD623. Presumably ACP-L is needed to accept propionate from AT-L to allow efficient synthesis of DEB.

A related experiment using pATD624, an equivalent plasmid that lacks the 107 aa segment, did not increase antibiotic production in the DEBS1-M1 strain. Indeed, deletion of just the 107 aa segment in the wild-type strain causes a loss of normal erythromycin biosynthesis (data not shown). This seems to indicate that the 107 aa segment is important for the activity of AT-L. We have detected a similar segment using BLAST searching (Altschul et al., 1990) upstream of ATs from other type I PKSs, including the remaining ATs of the erythromycin PKS, the rapamycin PKS in Streptomyces hygroscopicus (Schwecke et al., 1995), the oleandomycin PKS from S. antibioticus (Swan et al., 1994), a PKS-like cluster of S. ambofaciens (Aigle et al., 1996), mycocerosic acid synthase, a fatty acid elongating multifunctional enzyme from Mycobacterium tuberculosis (Mathur \& Kolattukudy, 1992), and phenylalanine ammonia-lyases from plants (Osakabe et al., 1995). Further work on the structure of this enzymic domain should reveal the relevance of the 107 aa segment to the activity of AT-L. Up to now most erythromycin derivatives have been made by chemical modifications to the antibiotic, but these techniques can be limited. Recent efforts, however, show that erythromycin derivatives can been produced by genetically reprogramming erythromycin-producing strains (McAlpine et al., 1987; Weber et al., 1991; Donadio et al., 1991, 1993; Jacoben et al., 1997; Ruan et al., 1997b). A better understanding of the structure and function of type I PKSs should enable the design of molecules with increased diversity and biological function.

\section{ACKNOWLEDGEMENTS}

We thank T. Kavanaugh, L. Zulawinski and S. Nannapeneni for synthesis of oligonucleotides. Also, we thank S. Kakavas for providing the nidKS1 sequence before publication.

\section{REFERENCES}

Aigle, B., Schneider, D., Morilhat, C., Vandewiele, D., Daty, A., Holl, A.-C., Simonet, J.-M. \& Decaris, B. (1996). An amplifiable and deletable locus of Streptomyces ambofaciens RP181110 contains a very large gene homologous to polyketide synthase genes. Microbiology 142, 2815-2824.

Altschul, S. F., Gish, W., Miller, W., Myers, E. W. \& Lipman, D. J. (1990). Basic local alignment search tool. J Mol Biol 215, 403-410.

Aparicio, J. F., Caffrey, P., Marsden, A. F. A., Stauton, J. \& Leadlay, P. F. (1994). Limited proteolysis and active-site studies of the first multienzyme component of the erythromycin-producing polyketide synthase. J Biol Chem 269, 8524-8528.
Bedford, D., Jacobsen, J. R., Luo, G., Cane, D. E. \& Khosla, C. (1996). A functional chimeric modular polyketide synthase generated via domain replacement. Chem Biol 3, 827-831.

Bevitt, D. J, Cortes, J., Haydock, S. F. \& Leadlay, P. F. (1992). 6-Deoxyerythronolide-B synthase 2 from Saccharopolyspora erythraea. Cloning of the structural gene, sequence analysis and inferred domain structure of the multifunctional enzyme. Eur $J$ Biochem 204, 39-49.

Bibb, M. J., White, J., Ward, J. M. \& Janssen, G. R. (1994). The mRNA for the $23 \mathrm{~S}$ rRNA methylase encoded by the ermE gene of Saccharopolyspora erythraea is translated in the absence of a conventional ribosome-binding site. Mol Microbiol 14, 533-545.

Caffrey, P., Bevitt, D. J., Staunton, J. \& Leadlay, P. F. (1992). Identification of DEBS1, DEBS2 and DEBS3, the multienzyme polypeptides of the erythromycin-producing polyketide synthase from Saccharopolyspora erythraea. FEBS Lett 304, 225-228.

Cortes, J., Haydock, S. F., Roberts, G. A., Bevitt, D. J. \& Leadlay, P. F. (1990). An unusually large multifunctional polypeptide in the erythromycin-producing polyketide synthase of Saccharopolyspora erythraea. Nature 348, 176-178.

Devereux, J., Haeberli, P. \& Smithies, 0 . (1984). A comprehensive set of sequence analysis programs for the VAX. Nucleic Acids Res 12, 387-395.

Dewitt, J. P. (1985). Evidence for a sex factor in Streptomyces erythreus. J Bacteriol 164, 969-971.

Donadio, S. \& Katz, L. (1992). Organization of the enzymatic domains in the multifunctional polyketide synthase involved in erythromycin formation in Saccharopolyspora erythraea. Gene 111, 51-60.

Donadio, S., Staver, M., McAlpine, J. B., Swanson, S. J. \& Katz, L. (1991). Modular organization of genes required for complex polyketide biosynthesis. Science 252, 675-679.

Donadio, S., McAlpine, J. B., Shelden, P. J., Jackson, M. \& Katz, L. (1993). An erythromycin analog produced by reprogramming polyketide synthesis. Proc Natl Acad Sci USA 90, 7119-7123.

Hopwood, D. A. \& Sherman, D. H. (1990). Molecular genetics of polyketides and its comparison to fatty acid biosynthesis. Annu Rev Genet 24, 37-66.

Hopwood, D. A., Bibb, M. J., Chater, K. F., Kieser, T., Bruton, C. J., Kieser, H. M., Lydiate, D. J., Smith, C. P., Ward, J. M. \& Schempf, H. (1985). Genetic Manipulation of Streptomyces: a Laboratory Manual. Norwich: John Innes Foundation.

Hsieh, Y.-J. \& Kolattukudy, P. E. (1994). Inhibition of erythromycin synthesis by disruption of malonyl-coenzyme A decarboxylase gene eryM in Saccharopolyspora erythraea. $J$ Bacteriol 176, 714-724.

Hutchinson, C. R. \& Fujii, I. (1995). Polyketide synthase gene manipulation: a structure-function approach in engineering novel antibiotics. Annu Rev Microbiol 49, 201-238.

Jacoben, J. R., Hutchinson, C. R., Cane, D. E. \& Khosla, C. (1997). Precursor-directed biosynthesis of erythromycin analogs by an engineered polyketide synthase. Science 277 , 367-369.

Kao, C. M., Katz, L. \& Khosla, C. (1994). Engineered biosynthesis of a complete macrolactone in a heterologous host. Science 265, 509-512.

Katz, L. \& Donadio, S. (1993). Polyketide synthesis: prospect for hybrid antibiotics. Annu Rev Microbiol 47, 875-912.

Kuhstoss, S., Huber, M., Turner, J. R., Paschal, J. W. \& Rao, N. (1996). Production of a novel polyketide through the construction of a hybrid polyketide synthase. Gene 183, 231-236.

McAlpine, J. B., Tuan, J. S., Brown, D. P., Grebner, K. D., Whittern, 
D. N., Buko, A. \& Katz, L. (1987). New antibiotics from genetically engineered actinomycetes. J Antibiot 40, 1115-1122.

Marsden, A. F. A., Caffrey, P., Aparicio, J. F., Loughran, M. S., Staunton, J. \& Leadlay, P. F. (1994). Stereospecific acyl transfers on the erythromycin-producing polyketide synthase. Science 263, 378-380.

Mathur, M. \& Kolattukudy, P. E. (1992). Molecular cloning and sequencing of the gene for mycocerosic acid synthase, a novel fatty acid elongating multifunctional enzyme, from $\mathrm{Myco}$ bacterium tuberculosis var. bovis Bacillus Calmette-Guerin. J Biol Chem 267, 19388-19395.

Molnar, I., Aparicio, J. F., Haydock, S. F., Khaw, L. E., Schwecke, T. \& O'Hagan, D. (1993). Biosynthesis of fatty acid and polyketide metabolites. Nat Prod Rep 10, 593-624.

Olinynyk, I., Brown, M. J. B., Cortes, J., Staunton, J. \& Leadlay, P. F. (1996). A hybrid modular polyketide synthase obtained by domain swapping. Chem Biol 3, 833-839.

Osakabe, Y., Osakabe, K., Kawai, S., Katayama, Y. \& Morohoshi, N. (1995). Characterization of the structure and determination of mRNA levels of the phenylalanine ammonia-lyase gene family from Populus kitakamiensis. Plant Mol Biol 28, 1133-1141.

Pieper, R., Ebert-Khosla, S., Cane, D. \& Khosla, C. (1996a). Erythromycin biosynthesis: kinetic studies on a fully active modular polyketide synthase using natural and unnatural substrates. Biochemistry 35, 2054-2060.

Pieper, R., Kao, C., Khosla, C., Luo, G. \& Cane, D. E. (1996b). Specificity and versatility in erythromycin biosynthesis. Chem Soc Rev 25, 297-302.

Ruan, X., Stassi, D., Lax, S. \& Katz, L. (1997a). A second type-I PKS gene cluster isolated from Streptomyces bygroscopicus ATCC 29253, a rapamycin-producing strain. Gene 184, 1-9.

Ruan, X., Pereda, A., Stassi, D., Zeidner, D., Summers, R. G., Jackson, M., Shivakumar, A., Kakavas, S., Staver, M. J., Donadio, S. \& Katz, L. (1997b). Acyltransferase domain substitutions in the erythromycin polyketide synthase yield novel erythromycin derivatives. J Bacteriol 179, 6416-6425.
Ruby, C. L. \& Danis, S. J. (1992). Complex organization of the Streptomyces avermitilis genes encoding the avermectin polyketide synthase. Gene 115, 119-125.

Sambrook, J., Fritsch, E. F. \& Maniatis, T. (1989). Molecular Cloning: a Laboratory Manual, 2nd edn. Cold Spring Harbor, NY: Cold Spring Harbor Laboratory.

Schwecke, T., Aparicio, J. F., Molnar, I., Konig, A., Khaw, L. E., Haydock, S. F., Oliynyk, M., Caffrey, P., Cortes, J. B., Lester, J. B., Bohm, G. A., Staunton, J. \& Leadlay, P. F. (1995). The biosynthethic gene cluster for the polyketide immunosuppressant rapamycin. Proc Natl Acad Sci USA 92, 7839-7843.

Swan, D. G., Rodriguez, A. M., Vilches, C., Mendez, C. \& Salas, J. A. (1994). Characterization of a Streptomyces antibioticus gene encoding a type I polyketide synthase which has an unusual coding sequence. Mol Gen Genet 242, 358-362.

Tsukamoto, N., Chuck, J.-A., Luo, G., Kao, C. M., Khosla, C. \& Cane, D. E. (1996). 6-Deoxyerythronolide B synthase 1 is specifically acylated by a diketide intermediate at the betaketoacyl-acyl carrier protein synthase domain of module 2 . Biochemistry 35, 15244-15248.

Vara, J. A., Lewandowska-Skarbek, M., Wang, Y.-G., Donadio, S. \& Hutchinson, C. R. (1989). Cloning of genes governing the deoxysugar portion of the erythromycin biosynthesis pathway in Saccharopolyspora erythraea (Streptomyces erythreus). $J$ Bacteriol 171, 5872-5881.

Weber, J. M. \& Losick, R. (1988). The use of a chromosome integration vector to map erythromycin resistance and production genes in Saccharopolyspora erythraea (Streptomyces erythreus). Gene 68, 173-180.

Weber, J. M., Leung, J. O., Swanson, S. J., Idler, K. B. \& McAlpine, J. B. (1991). An erythromycin derivative produced by targeted gene disruption in Saccharopolyspora erythraea. Science 252, 114-117.

Yamamoto, H., Maurer, K. H. \& Hutchinson, C. R. (1986). Transformation of Streptomyces erythreus. J Antibiot 39, 1304-1313.

Received 24 July 1997; revised 7 October 1997; accepted 8 October 1997. 